\title{
Recursive Approach for Evaluation of Time Intervals between Transac- tions in Polling Procedure
}

\author{
Eugene Larkin ${ }^{1}$, Alexey Ivutin ${ }^{2}$, Dmitry Esikov² \\ ${ }^{1}$ Tula State University, Department of Robotics and Industry Mechanization, Tula, Russia \\ ${ }^{2}$ Tula State University, Department of Computer Technology, Tula, Russia
}

\begin{abstract}
An ergodic semi-Markov process with the structure represented by the full graph with loops, which simulates a digital control algorithm that generated transactions onto an object, is investigated. Elementary simplifications for reduction of semi-Markov processes are defined. Recursive procedure for reduction of initial semi-Markov process structure till the model, which includes selected states with its links only, is proposed. Formulae for recalculation of probabilities, weighted densities and expectations of time of switching to linked states are obtained. It is shown that recursive procedure may be used also for calculation of time expectation of return the process to one of selected states that simplified the task of evaluation of time intervals between transactions in polling procedure.

Keywords. Polling, semi-Markov process, recursive procedure, expectation, dispersion, time interval.
\end{abstract}

\section{Introduction}

Polling is one of the most common procedure for transactions organization in real time, embedded, swarm etc., control systems $[1,2]$. In such a systems control computer algorithmically organizes a cycle of control of peripheral equipment, so there are rigid restrictions for time period between transactions.

For evaluation of time intervals the model of data processing must be worked out. Taking into account the number of specific features of control algorithms, such as cyclic recurrence, quasi-stochastic way of continuation in places of branching, quasi-probabilistic of time of execution of operators, there is the only formalism for modeling the computation, ergodic semiMarkov process [3-6], and interpretation of algorithm may be represented as a wandering through states of semi-Markov process [7, 8].

In $[9,10]$ matrix expressions for evaluation of time intervals were obtained, but they are cumbersome enough for practice use. So, the known approach needs high computational resources, commensurable with compilation of program and experimental evaluation of time intervals under consideration $[11,12]$. Recursive procedure proposed below permits substantially decrease time complexity of analysis of ergodic semiMarkov processes.

\section{Initial semi-Markov process}

In most general case semi-Markov process, which simulates a polling generator of transactions, is the next [36]:

$$
{ }^{S+J} M=\left\{{ }^{++J} A,{ }^{S+J} \boldsymbol{r},{ }^{S+J} \boldsymbol{h}(t)\right\},
$$

where ${ }^{S+J} A$ - is the set of states; ${ }^{S+J} A={ }^{S} A \cup{ }^{\bar{S}} A$; ${ }^{S} A \cap{ }^{\bar{S}} A=\varnothing ;{ }^{S} A=\left\{a_{1}, \ldots, a_{s}, \ldots, a_{S}\right\}$ - is the subset of selected states, which simulate operators of generation of transactions; ${ }^{\bar{S}} A=\left\{a_{S+1}, \ldots, a_{S+j}, \ldots, a_{S+J}\right\}-$ is the subset of other states; ${ }^{S+J} \boldsymbol{r}=\left({ }^{S+J} r_{m n}\right)$ - is the adjacency matrix of size $(S+J) \times(S+J) ;{ }^{S+J} \boldsymbol{h}(t)$ - is the semi-Markov matrix of size $(S+J) \times(S+J)$; $t$ - time;

$$
{ }^{S+J} \boldsymbol{h}(t)=\left[{ }^{S+J} \boldsymbol{p} \otimes{ }^{S+J} \boldsymbol{f}(t)\right] ;
$$

${ }^{S+J} \boldsymbol{p}$ - is the stochastic marix; ${ }^{S+J} \boldsymbol{f}(t)$ - is the matrix of densities which specifies time intervals; ${ }^{S+J} r_{m n}=1$ for $1 \leq m, n \leq J+S$.

Typical graph [13-14] which describes structure of process is shown on figure 1.

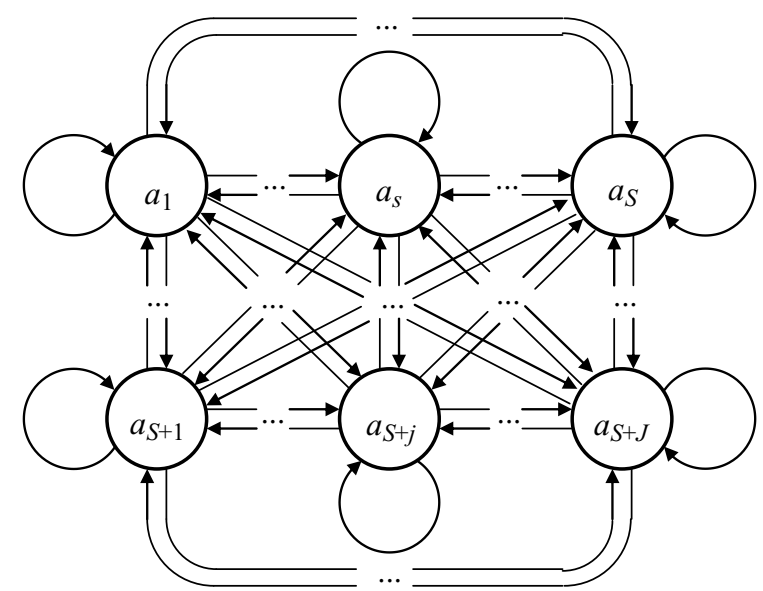

Figure 1. Structure of semi-Markov process 
The process (1) generates a transaction in one of the next cases:

1) when the direct switch from $a_{m} \in{ }^{S} A$ to $a_{n} \in{ }^{S} A$ occurs;

2) when occurs switch from $a_{m} \in{ }^{S} A$ to $a_{k} \in \bar{S}^{\bar{S}} A$ and subsequent wandering through states of subset $\bar{S}_{A}$ from $a_{k} \in^{\bar{S}} A$ to $a_{n} \in{ }^{S} A$.

For evaluation of time intervals it is necessary to replace the walk through states of subset ${ }^{\bar{S}} A$ to direct switch, i.e. reduce (1) till the semi-Markov process

$$
{ }^{S} M=\left\{{ }^{S} A,{ }^{S} \boldsymbol{r},{ }^{S} \boldsymbol{h}(t)\right\},
$$

where ${ }^{S} \boldsymbol{r}=\left({ }^{S} r_{m n}\right)$ - is the adjacency matrix of size $S \times S ;{ }^{S} r_{m n}=1,1 \leq m, n \leq S ;{ }^{S} \boldsymbol{h}(t)$ - is the semiMarkov matrix of size $S \times S$.

When reducing, states of subset ${ }^{\bar{S}} A$ are eliminated, so every switch of semi-Markov process (3) generates one transaction. For the reducing may be used matrix method, described in [10], however similar result may be obtained with the aid of recursive procedure of simplification of (1).

\section{Elementary operations of reducing}

For realization of recursive procedure let us to introduce three elementary operations of reducing of semiMarkov process, which are shown on figure 2 [15-16].
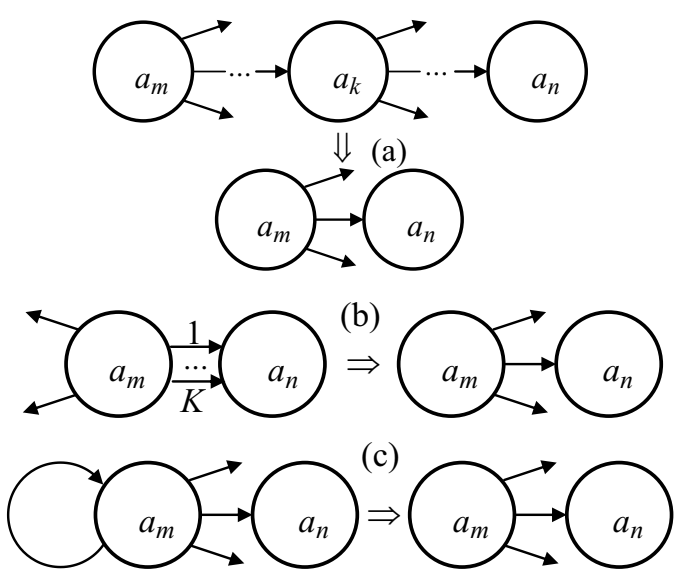

Figure 2. Operations of reducing

On the fig. 2 the union of sequential states is shown. Probability, weighted density, expectation and dispersion of time of wandering from $a_{m}$ to $a_{n}$ are determined by the next dependencies:

$$
\begin{gathered}
\widetilde{p}_{m, n}=\prod_{k=m}^{n-1} p_{k, k+1} \\
\widetilde{h}_{m, n}(t)=L^{-1}\left[\prod_{k=m}^{n-1} L\left[h_{k, k+1}(t)\right]\right] \\
\widetilde{T}_{m, n}=\sum_{k=m}^{n-1} T_{k, k+1}, \\
\widetilde{D}_{m, n}=\sum_{k=m}^{n-1} D_{k, k+1}
\end{gathered}
$$

where $p_{k, k+1}$ - is the probability of switch to linked state; $h_{k, k+1}(t)$ - weighted density of time of switching to linked state; $T_{k, k+1}$ - is the expectation of time of switching; $L[.],. L^{-1}[.$.$] - are direct and inverse La-$ place transforms.

On the fig. 2 (b) the union of parallel arcs is shown. Probability, weighted density, expectation and dispersion of time of switching from $a_{m}$ to $a_{n}$ are determined by the next dependencies:

$$
\begin{gathered}
\widetilde{p}_{m, n}=\sum_{k=1}^{K} p_{m, n, k} ; \\
\widetilde{h}_{m, n}(t)=\sum_{k=1}^{K} h_{m, n, k}(t) ; \\
\widetilde{T}_{m, n}=\frac{\sum_{k=1}^{K} p_{m, n, k} \cdot T_{m, n, k}}{\widetilde{p}_{m, n}}, \\
\widetilde{D}_{m, n}=\frac{\sum_{k=1}^{K} p_{m, n, k} \cdot\left(D_{m, n, k}+T_{m, n, k}^{2}\right)}{\widetilde{p}_{m, n}}-\widetilde{T}_{m, n}^{2}
\end{gathered}
$$

where $k$ - is the number of arcs from $a_{m}$ to $a_{n} ; p_{m, n, k}$, $h_{m, n, k}(t), T_{m, n, k}, D_{m, n, k}-$

probability, weighted density and expectation of time of switching from $a_{m}$ to $a_{n}$ on $k$-th arc.

On the fig. 2 (c) elimination of loop is shown. Probability, weighted density, expectation and dispersion of time of switching from $a_{m}$ to $a_{n}$ are determined by the next dependencies:

$$
\begin{gathered}
\tilde{p}_{m, n}=\frac{p_{m, n}}{1+p_{m, m}} ; \\
\widetilde{h}_{m, n}(t)=L^{-1}\left[\boldsymbol{I}_{r} \cdot \sum_{k=1}^{\infty}\left\{L\left[\boldsymbol{h}_{c}(t)\right]\right\}^{k} \cdot \boldsymbol{I}_{c}\right] ; \\
\boldsymbol{h}_{c}=\left(\begin{array}{cc}
h_{m, m}(t) & h_{m, n}(t) \\
0 & 0
\end{array}\right) ; \boldsymbol{I}_{c}=\left(\begin{array}{l}
0 \\
1
\end{array}\right) ; \boldsymbol{I}_{r}=(0,1) ; \\
\widetilde{T}_{m, n}=T_{m, n}+\frac{T_{m, m} \cdot p_{m, m}}{1-p_{m, m}} ; \\
\widetilde{D}_{m, n}=D_{m, n}+\frac{D_{m, m} \cdot p_{m, m}}{1-p_{m, m}}+\frac{T_{m, m}^{2} \cdot p_{m, m}}{\left(1-p_{m, m}\right)^{2}} .
\end{gathered}
$$

\section{The recursive procedure}

The recursive procedure presupposes sequential elimination of states from $(S+J)$-th, to $(S+1)$-th. Let us assume that at the previous simplifications states from $(S+J)$-th till $(S+1+1)$-e were eliminated, i.e. semiMarkov process (1) was reduced till the process

${ }^{S+j} M=\left\{{ }^{S+j} A,{ }^{S+j} \boldsymbol{r},{ }^{S+j} \boldsymbol{h}(t)\right\}$,
where ${ }^{S+j} \boldsymbol{r}=\left({ }^{S+j} r_{m n}\right)$ and ${ }^{S+J} \boldsymbol{h}(t)=\left[{ }^{S+J} h_{m n}(t)\right]$ are of size $S+j \times S+j ;{ }^{S} r_{m n}=1,1 \leq m, n \leq S+j$.

The first operation of reducing is elimination of loop on the state $a_{S+j}$ due to dependencies (6): 


$$
\begin{aligned}
& { }^{S+j} \widetilde{p}_{S+j, n}=\frac{{ }^{S+j} p_{S+j, n}}{1+{ }^{S+j} p_{S+j, S+j}} ; \\
& \left.\tilde{h}_{S+j, n}(t)=L^{-1}\left[\boldsymbol{I}_{r} \cdot \sum_{k=1}^{\infty}\left\{L^{S+j} \boldsymbol{h}_{c}(t)\right]\right]^{k} \cdot \boldsymbol{I}_{c}\right] \text {; } \\
& { }^{S+j} \boldsymbol{h}_{c}=\left(\begin{array}{cc}
{ }^{S+j} h_{S+j, S+j}(t) & { }^{S+j} h_{S+j, n}(t) \\
0 & 0
\end{array}\right) ; \\
& { }^{S+j} \widetilde{T}_{S+j, n}={ }^{S+j} T_{S+j, n}+\frac{{ }^{S+j} T_{S+j, S+j} \cdot{ }^{S+j} p_{S+j, S+j}}{1-{ }^{S+j} p_{S+j, S+j}} ; \\
& { }^{S+j} \widetilde{D}_{S+j, n}={ }^{S+j} D_{S+j, n}+\frac{{ }^{S+j} D_{S+j, S+j} \cdot{ }^{S+j} p_{S+j, S+j}}{1-{ }^{S+j} p_{S+j, S+j}}+ \\
& +\frac{{ }^{S+j} T_{S+j, S+j}^{2} \cdot{ }^{S+j} p_{S+j, S+j}}{\left(1-{ }^{S+j} p_{S+j, S+j}\right)^{2}},
\end{aligned}
$$

The next operation of the recursive cycle is splitting the state $a_{S+j}$ onto $(S+j-1)^{2}$ states as it is shown on fig. 3 .

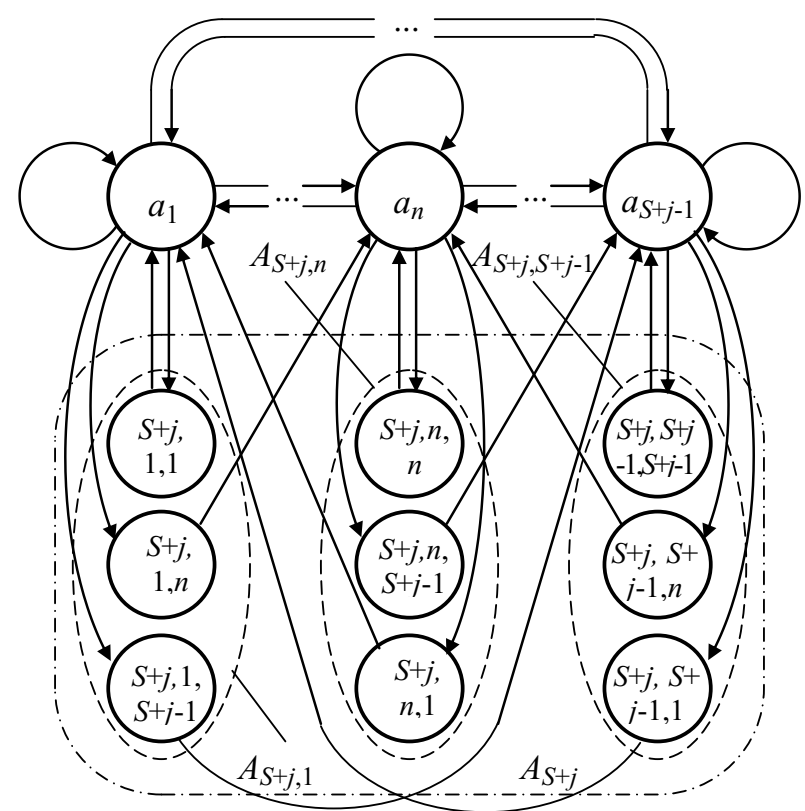

Figure 3. Splitting the state $a_{S+j}$

At first the state $a_{S+j}$ (is circled with dash-dotted line) is splitting onto set $A_{S+j}=\left\{a_{S+j, 1}, \ldots, a_{S+j, n}, \ldots, a_{S-j, S+j-1}\right\}$ (every element of set is circled with dashed line), and then states $a_{S+j, n}, 1 \leq n \leq S+j-1$ are splitting onto sets $A_{S+j, n}=\left\{a_{S+j, n, 1}, \ldots, a_{S+j, n, n}, \ldots, a_{S-j, n, S+j-1}\right\}$. In other to ensure equivalency of transforms probabilities, weighted densities and expectations must be recalculated in accordance with dependencies (4):

$$
\begin{gathered}
\hat{p}_{n, m}={ }^{S+j} p_{n, S+j}{ }^{S+j} \widetilde{p}_{S+j, m} ; \\
\hat{h}_{n, m}(t)=L^{-1}\left[L\left[L^{S+j} h_{n, S+J}(t)\right] \cdot L^{[S+j} \widetilde{h}_{S+J, m}(t)\right] ; \\
\hat{T}_{n, m}(t)={ }^{S+j} T_{n, S+J}+{ }^{S+j} \widetilde{T}_{S+J, m} ;
\end{gathered}
$$

$$
\begin{aligned}
\hat{D}_{n, m}(t) & ={ }^{S+j} D_{n, S+J}+{ }^{S+j} \widetilde{D}_{S+J, m}, \\
1 & \leq n, m \leq S+j-1
\end{aligned}
$$

On the next step in accordance with (5) must be recalculated probabilities, weighted densities and expectations of semi-Markov process ${ }^{S+j-1} M=\left\{{ }^{S+j-1} A,{ }^{S+j-1} \boldsymbol{r},{ }^{S+j-1} \boldsymbol{h}(t)\right\}$, which have already $S+j-1$ states.

$$
\begin{gathered}
{ }^{S+j-1} p_{n, m}={ }^{S+j} p_{n, m}+\hat{p}_{n, m} ; \\
{ }^{S+j-1} h_{n, m}={ }^{S+j} h_{n, m}(t)+\hat{h}_{n, m}(t) ; \\
{ }^{S+j-1} T_{n, m}=\frac{{ }^{+j} p_{n, m} \cdot{ }^{S+j} T_{n, m}+\hat{p}_{n, m} \cdot \hat{T}_{n, m}}{{ }^{S+j} p_{n, m}+\hat{p}_{n, m}} ; \\
\left.+\frac{{ }^{S+j} p_{n, m} \cdot\left({ }^{S+j-1} D_{n, m}=-{ }^{S+j-1} T_{n, m}^{2}+\right.}{}+{ }^{S+j} T_{n, m}^{2}\right)+\hat{p}_{n, m}\left(\hat{D}_{n, m}+\hat{T}_{n, m}^{2}\right) \\
{ }^{S+j} p_{n, m}+\hat{p}_{n, m}
\end{gathered} .
$$

Recursion procedure repeated from $j=J$ till $j=1$. As a result of such a procedure elements of matrices ${ }^{S} \boldsymbol{p}=\left[{ }^{S} p_{m n}\right\rfloor,{ }^{S} \boldsymbol{h}=\left[{ }^{S} h_{m n}(t)\right]$ and ${ }^{S} \boldsymbol{T}=\left({ }^{S} T_{m n}\right)$ are calculated for the semi-Markov process (3).

\section{Time intervals between transactions}

Process (3) still stay the ergodic one, so density of time intervals between transactions may be defined in accordance with $[3,4]$ the next expression:

$$
g(t)=\sum_{m=1}^{S} \frac{\sum_{n=1}^{S} S p_{m, n}{ }^{S} T_{m, n}}{{ }^{1} T_{m, m}}\left[\sum_{n=1}^{S}{ }^{S} h_{m, n}\right],
$$

where $\sum_{n=1}^{S}{ }^{S} h_{m, n}$ and $\sum_{n=1}^{S} S p_{m, n}{ }^{S} T_{m, n}$ - density and expectation of time of stay of semi-Markov process (3) in the state $a_{m} \in{ }^{S} A ;{ }^{1} T_{m, m}$ - expectation of returning of the ergodic process (3) to state $a_{m}$.

For determining ${ }^{1} T_{m, m}$ one may use recursive procedure, described above. Without loss of community let us describe it for determining ${ }^{1} T_{1,1}$. For other nominations of $1 \leq m \leq S$ procedure may be applied to process (3) after a changing of numeration of states of the process.

Procedure for decrement of $m$, from $S$ to 1 , is the next:

the elimination of loop

$$
{ }^{o} \widetilde{p}_{m, n}=\frac{{ }^{m} p_{m, n}}{1+{ }^{m} p_{m, m}} ;{ }^{m} \widetilde{T}_{m, n}={ }^{m} T_{m, n}+\frac{{ }^{m} T_{m, m} \cdot{ }^{m} p_{m, m}}{1-{ }^{m} p_{m, m}} ;
$$

the splitting of set and union of sequential states

$$
\begin{gathered}
\hat{p}_{n, k}={ }^{m} p_{n, m} \cdot{ }^{m} \widetilde{p}_{m, k} ; \hat{T}_{n, k}(t)={ }^{m} T_{n, m}+{ }^{m} \widetilde{T}_{m, k} ; \\
1 \leq n, k \leq m-1 ;
\end{gathered}
$$

union of parallel arcs

$$
\begin{gathered}
{ }^{m-1} p_{n, k}={ }^{m} p_{n, k}+\hat{p}_{n, k} ; \\
{ }^{m-1} T_{n, k}=\frac{{ }^{m} p_{n, k} \cdot{ }^{m} T_{n, k}+\hat{p}_{n, k} \cdot \hat{T}_{n, k}}{{ }^{m-1} p_{n, k}} .
\end{gathered}
$$


Procedure must be repeated for different states, which must be placed onto the first position to achieve all parameters necessary to use the formula (10).

\section{Experimental verification of method}

For verification of proposed method direct computer experiment was executed with use the Monte-Carlo method. Under verification was homogeneous semiMarkov process ${ }^{3} M=\left\{{ }^{3} A,{ }^{3} \boldsymbol{r},{ }^{3} \boldsymbol{h}(t)\right\}$ which is shown on figure 4.

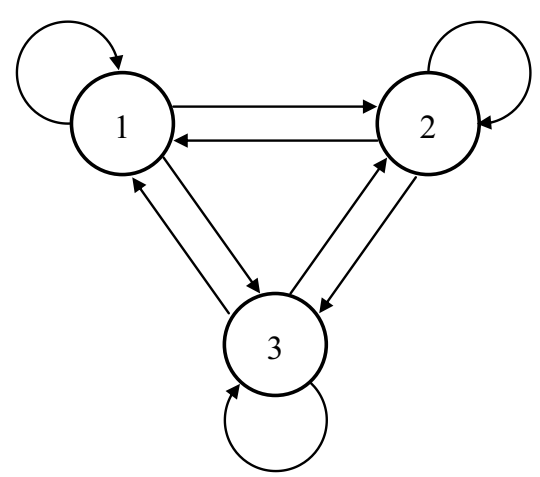

Figure 4. Semi-Markov process ${ }^{3} M$

In semi-Markov process ${ }^{3} M$ :

$$
\begin{aligned}
& { }^{3} A=\{1,2,3\} ; \\
& { }^{3} \boldsymbol{r}=\left(\begin{array}{lll}
1 & 1 & 1 \\
1 & 1 & 1 \\
1 & 1 & 1
\end{array}\right) ; \\
& { }^{3} \boldsymbol{h}(t)=\left[\begin{array}{lll}
1 / 3 \cdot f(t) & 1 / 3 \cdot f(t) & 1 / 3 \cdot f(t) \\
1 / 3 \cdot f(t) & 1 / 3 \cdot f(t) & 1 / 3 \cdot f(t) \\
1 / 3 \cdot f(t) & 1 / 3 \cdot f(t) & 1 / 3 \cdot f(t)
\end{array}\right] \\
& f(t)=\left\{\begin{array}{l}
5 / 3, \text { if } 0,7 \leq t \leq 1,3 ; \\
0, \text { if } t<0,7, \text { or } t>1,3 .
\end{array}\right.
\end{aligned}
$$

Expectation and dispersion of $f(t)$ are equal $T_{m, n}=1 s, D_{m, n}=0,03 s^{2}, 1 \leq m, n \leq 3$.

Calculation with use the proposed method gives the following result: ${ }^{1} T_{1,1}=3 \mathrm{~s} ;{ }^{1} D_{1,1}=6,123 \mathrm{~s}^{2}$.

Computer experiment was carried out in accordance with the next classical method.

1) Reset the counter of number of realizations, $l=0$.

2) Assignment the status of current to the first state, $a_{l}=1$.

3) Reset the timer $t_{l}=0$.

4) Receiving a random value $1 \leq \pi \leq 1$ with uniform distribution.

5) If $0 \leq \pi \leq 0,333$, then $a_{l}=1$; if $0,333<\pi \leq 0,666$, then $a_{l}=2$; if $0,666<\pi \leq 1$, then $a_{l}=3$.

6) Receiving a random value $1 \leq \pi \leq 1$.

7) Calculation of time increment on the formula $\Delta_{t}=0,6 \pi+0,7 s$

8) Calculation of current time $t_{l}:=t_{l}+\Delta_{t}$;

9) If $a_{l} \neq 1$, then 4 .
10) Unloading $t_{l}$ to array of results.

11) $l:=l+1$. If $l<L$, then 3 ).

12) End of experiment.

On figure 5 the histogram of time of returning to state 1 is shown, which was constructer after 10000 realizations of the process ${ }^{3} M$.

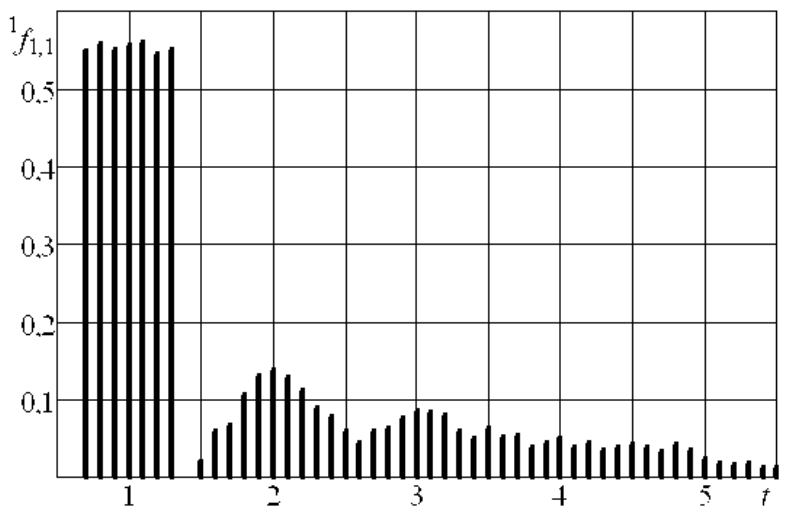

Figure 5. Histogram of ${ }^{1} f_{1,1}(t)$

Expectation and dispersion of density ${ }^{1} f_{1,1}(t)$, resulting from the processing of experimental data, are equal, correspondingly ${ }^{1} T_{1,1}=2,996 \mathrm{~s}$, with error $0,13 \%$, and $\sqrt{{ }^{1} D_{1,1}}=2,493$ with error $0,75 \%$. Experimental parameters rather precisely correspond to theoretical settlements.

\section{Conclusion}

In this article relatively simple universal procedure of recursive simplification of semi-Markov process was presented to determine time intervals between transactions at polling. Time characteristics were obtained for most common structure of semi-Markov generator of transactions (full graph with loops). Time intervals are significantly important parameter from point of view insertion the computer into control circuit as the feedback element. Optimization of time under consideration permits to improve quality characteristics of control. Further research in this area may be directed onto working out the method of optimization of time intervals with use recursive procedure.

\section{References}

1. Olsson, G., Piani, G. Computer Systems for Automation and Control (2000)

2. Ivutin, A., Larkin, E., Kotov, V. LNCS, 415 (2015)

3. Janssen, J. Semi-Markov models: theory and applications (2013)

4. Meerschaert, M., Straka, P. The Annals of Probability, 42, 1699 (2014)

5. Barbu V.S., Limnios N. Algebraic Methods in Statistics and Probability, 2, 19 (2010)

6. Barbu V.S., Limnios N. Lecture Notes in Statistics, 191 (2008) 
7. Howard, Ronald A. Dynamic Probabilistic Systems, Volume I: Markov Models. Vol. 1 (2012)

8. Howard, Ronald A. Dynamic Probabilistic Systems, Volume II: Semi-Markov and Decision Processes. Vol. 2 (2013)

9. Ivutin, A., Larkin, E. MECO, 236 (2014)

10. Ivutin, A., Larkin, E., Lutskov, Y. MECO, 250 (2015)

11. Zaroliagis, Ch., Grammati P., Kontogiannis S. Alg., Prob., Nets and Games, 9295 (2015)
12. Iverson, M., Ozguner, F., Follen, G. HPDC Proc. 263 (1996)

13. Godsil C., Royle G. Algebraic graph theory (2013)

14. Bollobas B. Modern graph theory (2013)

15. Beizer, B. Micro-Analysis of Computer System Performance (1978)

16. Ferrari, B., Computer Systems Performance Evaluation (1978) 